\title{
Statistical properties of giant pulses from the Crab pulsar
}

\author{
M. V. Popov ${ }^{1}$ and B. Stappers ${ }^{2}$ \\ 1 Astro Space Center of the Lebedev Physical Institute, Profsoyuznaya 84/32, Moscow, 117997 Russia \\ e-mail: mpopov@asc.rssi.ru \\ 2 Astronomical Institute "Anton Pannekoek", University of Amsterdam, Kruislaan 403, 1098 SJ Amsterdam; Stichting ASTRON, \\ Postbus 2, 7990 AA, Dwingeloo, The Netherlands
}

Received 18 October 2006 / Accepted 31 March 2007

\begin{abstract}
Aims. We have studied the statistics of giant pulses from the Crab pulsar for the first time with particular reference to their widths. Methods. We have analyzed data collected during $3.5 \mathrm{~h}$ of observations conducted with the Westerbork Synthesis Radio Telescope operated in a tied-array mode at a frequency of $1200 \mathrm{MHz}$. The PuMa pulsar backend provided voltage recording of X and Y linear polarization states in two conjugate $10 \mathrm{MHz}$ bands. We restricted the time resolution to $4 \mu \mathrm{s}$ to match the scattering on the interstellar inhomogeneities.

Results. In total about 18000 giant pulses (GP) were detected in full intensity with a threshold level of $6 \sigma$. We analyzed cumulative probability distribution (CPD) of giant pulse energies for groups of GPs with different effective widths in the range 4 to $65 \mu$ s. The CPDs were found to manifest notable differences for the different GP width groups. The slope of a power-law fit to the high-energy portion of the CPD evolves from -1.7 to -3.2 when going from the shortest to the longest GPs. There are breaks in the CPD powerlaw fits indicating flattening at low energies with indices varying from -1.0 to -1.9 for the short and long GPs, respectively. The GPs with a stronger peak flux density were found to be of shorter duration. We compare our results with previously published data and discuss the importance of these peculiarities in the statistical properties of GPs for the theoretical understanding of the emission mechanism responsible for GP generation.
\end{abstract}

Key words. stars: pulsars: general - stars: pulsars: individual: B0531+21 - methods: statistical - radiation mechanisms: non-thermal

\section{Introduction}

Giant pulses are one of the most striking phenomena of pulsar radio emission. Their flux density can exceed thousands of times the average pulse-integrated flux. Although recently there are reports of detecting excessively strong pulses from a number of pulsars (Johnston et al. 2001; Kramer et al. 2002; Romani \& Johnston 2001; Johnston \& Romani 2002; Kuzmin et al. 2004; Ershov \& Kuzmin 2003; Knight et al. 2006), only the Crab pulsar and the millisecond pulsar B1937+21 generate giant pulses numerous enough to study their statistical properties. The very large fluxes of giant pulses are coupled with an extremely short duration. Indeed, the overwhelming majority of giant pulses from the millisecond pulsar B1937+21 are shorter than $15 \mathrm{~ns}$ (Soglasnov et al. 2004), while giant pulses from the Crab pulsar have a mean width of about few microseconds (Hankins 2000) with occasional bursts shorter than 2 ns (Hankins et al. 2003).

The longitude position of giant pulses is remarkable in that it coincides with the position of high-energy emission (Moffett \& Hankins 1996; Cusumano et al. 2003; Johnston et al. 2004; Knight et al. 2006). Furthermore, giant pulses originate in a very narrow phase window that in general does not correspond to the phase window of regular radio emission. In the millisecond pulsar B1937+21, giant pulses are observed at the very trailing edge of the average profile in both the main pulse and the interpulse (Cognard et al. 1996; Kinkhabwala \& Thorsett 2000; Soglasnov et al. 2004). Popov et al. (2006) has recently suggested that giant pulses from the Crab pulsar are also seen at the trailing edge of the regular radio emission window, which they consider to be the precursor. In other words, they consider that radio emission in the main pulse and the interpulse of the Crab pulsar consists completely of giant pulses. Lastly, an essential property of giant pulses, by which they may be distinguished from normal strong pulses, is the distribution of their flux densities, which appears to follow a power law in contrast with the Gaussian or exponential flux distribution typical of regular (ordinary) individual pulses (Backer 1971; Hesse \& Wielebinski 1974; Ritchings 1976). In this paper we present an analysis of giant pulse-energy distribution based on the observations conducted with the Westerbork Synthesis Radio Telescope (WSRT) at $1200 \mathrm{MHz}$ using the PuMa (Voute et al. 2002) pulsar backend.

\section{Observations and data reduction}

The observations were carried out in November 2003 as part of a multi-frequency observing campaign that also included Jodrell Bank at $1420 \mathrm{MHz}$, Effelsberg at $8350 \mathrm{MHz}$, Kalyazin at $600 \mathrm{MHz}$, Pushchino at $111 \mathrm{MHz}$, and Kharkov (UTR-2) at $23 \mathrm{MHz}$. Simultaneous optical observations were also made with the 6-m telescope of the Special Astrophysical Observatory and 2.8-m telescope at La Palma. The MAGIC and HESS gamma-ray telescopes in La Palma and Namibiya also participated. While some results obtained at the separate observatories have already been published (Jessner et al. 2005; Popov et al. 2006), the joint analysis of multi-frequency observations will be presented in future publications.

We have analyzed the data obtained in observations with the WSRT during about 3.5 hours in two conjugate bands of $10 \mathrm{MHz}$ each at a central frequency of $1197 \mathrm{MHz}$. Baseband 
voltages of $\mathrm{X}$ and $\mathrm{Y}$ linear polarization states were recorded with two-bit sampling at Nyquist frequency. The coherent predetection dedispersion technique originally developed by Hankins (Hankins 1971; Hankins \& Rickett 1975) was used to remove dispersion smearing in the received pulsar signal. We took the value of dispersion measure $\left(56.757 \mathrm{pc} \mathrm{cm}^{-3}\right)$ and the timing model from the Jodrell Bank Monthly Ephemeris (Lyne 1982). The technique provides a formal time resolution of $100 \mathrm{~ns}$, but the expected pulse-broadening time due to interstellar scintillations is in the range 2 to $4 \mu \mathrm{s}$, based on the estimation made by Kuzmin et al. (2002). Therefore, we averaged the recorded signal after dispersion removal and square-low detection synchronously with a topocentric pulsar period into 8192 bins per period and with a resulting sampling interval close to $4.1 \mu \mathrm{s}$. The total intensity time series was finally formed and used for detection of giant pulses with the threshold level of $6 \sigma$. To provide better sensitivity for wider giant pulses, we developed a searching procedure which progressively tried to increase the averaging time interval $\left(\tau_{i}\right)$ by $1,2,3,4,6,8,12,16,24,32$, and 48 samples, i.e. from $4.1 \mu$ s to $196 \mu$ s. The averaging time with the best signal-to-noise ratio (SNR) was selected as $W_{\mathrm{e}}$ for the GP, detected simultaneously at several averaging times. The pulse energy $E$ (or average flux density) was calculated for the GP as a product of the SNR and the pulse width, equal to the averaging time, which corresponds to the best SNR value. In this approach we have different threshold levels both in peak flux density $F_{\mathrm{p}}$ and in pulse energy $E$ for each separate averaging time. The value of the root-mean-square deviation (rms or $\sigma$ ) goes down with increasing time averaging as the square root of time. Therefore, the threshold level in peak flux density $F_{\mathrm{p}}$ decreases with increasing averaging time as $F_{\mathrm{p}}(\tau) \propto 1 / \sqrt{\tau}$, while the threshold level in pulse energy $E$ increases with $\tau$ as $E(\tau) \propto \sqrt{\tau}$.

For the Crab pulsar, the system temperature is notably influenced by the impact of the Crab Nebula, whose flux density can be approximated by the relation $F_{v}=955 v^{-0.27} \mathrm{Jy}(v$ in $\mathrm{GHz})$ (Allen 1973; Bietenholz et al. 1997), which gives $F_{1200}=909 \mathrm{Jy}$ at $1200 \mathrm{MHz}$. In our observations we used all 14 dishes of the WSRT in tied-array mode, where all telescopes are added coherently, with the width of synthesized beam being equal to 34 arcsec, thus reducing the contribution from the Crab Nebula to the system noise by a factor $f_{v}=\Omega_{A} / \Omega_{\mathrm{CN}}=0.14$ to the value $F_{\mathrm{CN}}=127.6 \mathrm{Jy}$. Here $\Omega_{\mathrm{CN}}$ is a solid angle of the Crab Nebula, and $\Omega_{\mathrm{A}}$ a solid angle of the intersection of the synthesized beam with the Crab Nebula. However, there is still the contribution from the individual dishes; the system temperature of every 25-m dish was increased by the Crab Nebula emission by $\Delta T_{\mathrm{CN}}=F_{1200} G=86.3 \mathrm{~K}$, where $\mathrm{G}$ is the gain of a single telescope, equal to 0.095 at $1200 \mathrm{MHz}$. The intrinsic system temperature in the absence of the Crab Nebula is $30 \mathrm{~K}$ at $1200 \mathrm{MHz}$, and the resulting total system temperature is thus $116.3 \mathrm{~K}$, equivalent to $F_{\text {sys }}=87.4 \mathrm{Jy}$ for the gain in the tied array $(1.33 \mathrm{~K} / \mathrm{Jy})$, which when combined with the nebula contribution to the tied array becomes $F_{\text {tot }}=F_{\text {sys }}+F_{\mathrm{CN}}=215 \mathrm{Jy}$. One can see that WSRT reduces the contribution of the Crab Nebula to the system noise considerably when compared with the single dish observations at this frequency. This improvement will permit us to follow the statistics of giant pulses from the Crab pulsar to the lower energies; namely, the limiting rms $(\sigma)$ value in one polarization is $F_{\text {tot }} / \sqrt{B \tau}$, with $B=10 \mathrm{MHz}$, and $\tau=4.1 \mu \mathrm{s}$ the rms is equal to $33.5 \mathrm{Jy}$. For total intensity, the rms will be reduced by $\sqrt{2}$, and the threshold of $6 \sigma$ in peak flux density will be equal to $142 \mathrm{Jy}$. The threshold will be lower for averaged records, with a limiting

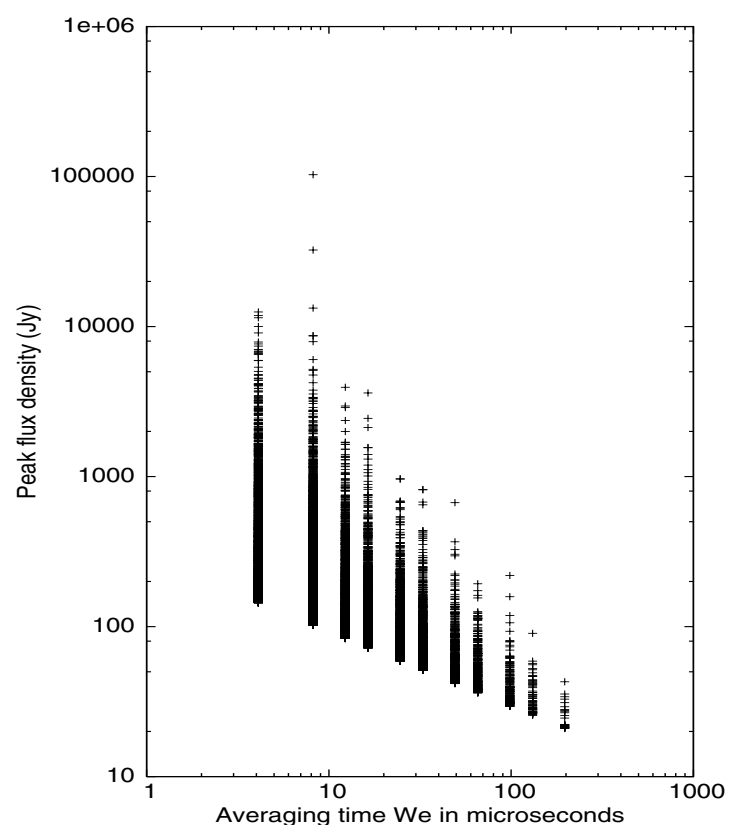

Fig. 1. Peak flux density versus the effective puse width $W_{\mathrm{e}}$ for all detected giant pulses.

value of $20 \mathrm{Jy}$ for giant pulses, which have a maximum effective width of about $200 \mu \mathrm{s}$.

In total 17869 giant pulses were detected over 370000 pulse periods with 14994 giant pulses located at the longitude of the main pulse and 2875 giant pulses associated with the interpulse. The background rate was determined by counting events at quiescent phases of the pulsar period and the corresponding values were taken into account in our statistical calculations. In fact, at the lowest range, between $6 \sigma$ and $7 \sigma$, only about $10 \%$ of all pulses we potentially incorrectly identified as giant pulses.

\section{Results}

Figure 1 represents the complete set of data showing peak flux density $F_{\mathrm{p}}$ versus the effective pulse width $W_{\mathrm{e}}$. The striped nature of the diagram is caused by the procedure of giant pulse detection, which is based on the discrete values of averaging time $W_{\mathrm{e}}$. The diagram demonstrates the peculiarity of the statistics on giant pulses: the strongest pulses clearly have a shorter duration; namely, there are no pulses with peak flux density greater than $1000 \mathrm{Jy}$ that are wider than $16 \mu \mathrm{s}$. As a consequence, giant pulses with different durations have different distributions in pulse energy, and the distribution in giant pulse width depends on the range of pulse energy used. Therefore, in our analysis, we separated the giant pulses belonging to the main pulse longitudes into five groups by their widths: (a) - GPs with the effective width $W_{\mathrm{e}}$ of $4.1 \mu \mathrm{s}$, (b) - GPs with the $W_{\mathrm{e}}$ of $8.2 \mu \mathrm{s}$, (c) - combined group of GPs with the $W_{\mathrm{e}}$ of 12.3 and $16.4 \mu \mathrm{s}$, (d) - combined group of GPs with the $W_{\mathrm{e}}$ of 24.5 and $32.8 \mu \mathrm{s}$, and (e) - combined group of GPs with the $W_{\mathrm{e}}$ of 49.2 and $65.6 \mu \mathrm{s}$. A separate group (f) was formed for the GPs detected at the longitude of the interpulse with the $W_{\mathrm{e}} 4.1,8.2$, and $12.3 \mu \mathrm{s}$. Then, pulse energy, or integrated flux density, was calculated as $E=F_{\mathrm{p}} \times W_{\mathrm{e}}$.

In our analysis we use cumulative probability distribution (CPD), which gives the number of pulses $N(E)$, above pulse energy $E$. Some authors prefer to calculate the probability distribution (PD) by giving the number of pulses $n(E)$ per interval 

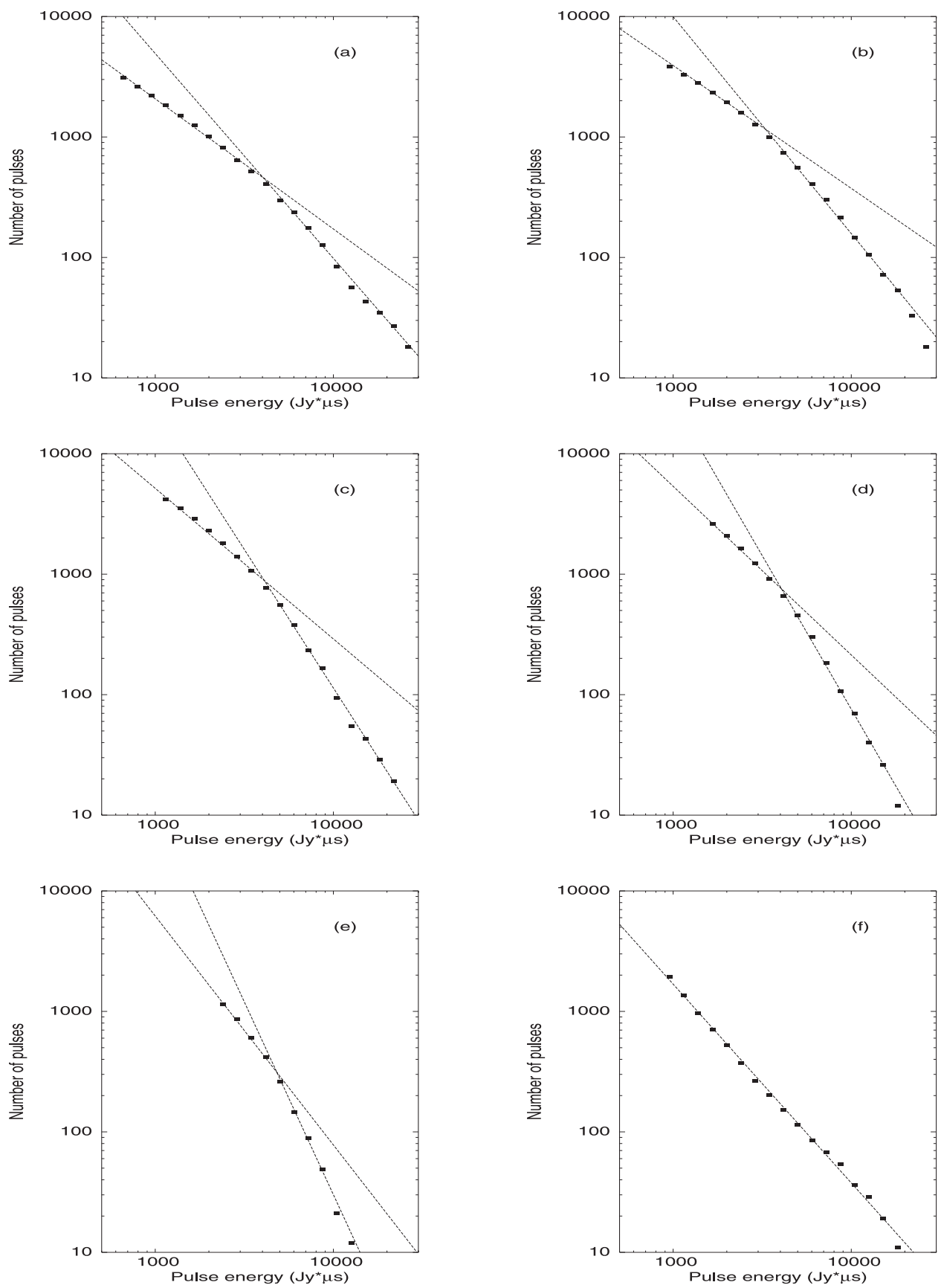

Fig. 2. The CPD of giant pulses with pulse energy above the displayed value for different groups of giant pulses classified by their effective width $W_{\mathrm{e}}$ as described in the Table 1 . Straight lines represent the fit by power-law functions with the parameters indicated in the Table 1 . The last plot (f) represents giant pulses belonging to the interpulse.

of energy $\mathrm{d} E$. If a power-law fit is used for the PD function as $n(E) \propto E^{-\beta}$, then a power-law fit will be also valid for the CPD function $N(E) \propto E^{-\gamma}$, since

$N\left(E>E_{y}\right)=\int_{E_{y}}^{\infty} n(E) \mathrm{d} E \propto E_{y}^{-\beta+1}$

with $\gamma=\beta-1$ in the absolute value.

Figure 2 displays the CPD of pulse energies for all the aforementioned groups of GPs. The CPD for the GPs belonging to the interpulse can be fitted with a power-law function with $\gamma=1.6 \pm 0.1$. The CPDs for the GPs in the main pulse manifest a break in the power-law index at certain values of pulse energy, which is slightly different for the different groups. Table 1 contains the results of a least-square solution for the power-law fit. The first column indicates the group of GPs, $W_{\mathrm{e}}$ is the averaging time for the group in $\mu \mathrm{s}, \gamma_{\text {high }}$ and $\gamma_{\text {low }}$ are indices of the power law function for the high energy tail and low energy portion of the CPD, respectively, and $E_{\text {break }}$ indicates the pulse energy where the slope of the power law changes. The break energy values were obtained as the crossing point between two straight lines defined by the least-square solutions. Relative inaccuracy is within $10 \%$ at the RMS level.

The absence of any break in the power-law fit for the CPD of the GPs belonging to the interpulse longitudes is good proof that the observed breaks in the CPDs of the main pulse GPs were not caused by selection effects, since we used exactly the same procedure for GPs detection irrespective of pulse longitude. A 
Table 1. The list of parameters of the power-law fits to the CPDs displayed in Fig. 2.

\begin{tabular}{ccccc}
\hline \hline Group & $W_{\mathrm{e}}(\mu \mathrm{s})$ & $\gamma_{\text {high }}$ & $\gamma_{\text {low }}$ & $E_{\text {break }}(\mathrm{Jy} \mu \mathrm{s})$ \\
\hline (a) & 4.1 & 1.7 & 1.1 & 4000 \\
(b) & 8.2 & 1.8 & 1.0 & 3500 \\
(c) & $12.3,16.4$ & 2.3 & 1.2 & 4000 \\
(d) & $24.5,32.8$ & 2.5 & 1.4 & 4000 \\
(e) & $49.2,65.6$ & 3.2 & 1.9 & 5000 \\
(f) & $4.1,8.2,12.3$ & 1.6 & 1.6 & \\
\hline
\end{tabular}

possible explanation is that we observe the regions where the GPs are generated in the the main pulse and the interpulse at different impact angles relative to the GP beam, which has a half width of about 5 degrees. Indeed, the comparison of the CPDs (combined for the width groups (a), (b), and (c)) for the main pulse and the interpulse shows that the CPDs would be identical if one were to multiply the interpulse GP energies by a factor of about 4 and increase their rate of occurrence by a factor of about 2. Therefore, the weakest detected interpulse GPs with a pulse energy of about $1000 \mathrm{Jy} \mu \mathrm{s}$, when observed at the same impact angle as the main pulse GPs (multiplied by 4), will have pulse energies of $4000 \mathrm{Jy} \mu \mathrm{s}$, which is above the break point.

\section{Discussion and conclusions}

The CPD function of the GPs originating at the longitudes of the main pulse manifests gradual changes in the power-law index from -1.7 to -3.2 at the high energy part of the CPD, and from -1.0 to -1.9 at the low energy portion of the CPD. As a rough guide, all GPs can be separated into two main groups: short GPs belonging to groups (a) and (b), and long GPs with an $W_{\mathrm{e}}$ greater than $8.1 \mu \mathrm{s}$. It is interesting to note that the CPD power-law index of about -1.7 for short GPs is close to the exponent determined for the CPD of the GPs from the millisecond pulsar $\mathrm{B} 1937+21$, which was found to be equal to -1.8 at $430 \mathrm{MHz}$ (Cognard et al. 1996). The value was confirmed by Kinkhabwala \& Thorsett (2000), and recently estimated by Soglasnov et al. (2004) as -1.4 at $1650 \mathrm{MHz}$. All GPs from the millisecond pulsar are very short, lasting only 1-2 $\mu$ s as measured by Kinkhabwala \& Thorsett (2000), while Soglasnov et al. (2004) have found that the majority of GPs from this pulsar are shorter than $15 \mathrm{~ns}$.

Argyle \& Gower (1972) were the first to present the CPD for GPs from the Crab pulsar. They combined the results of observations made at $146 \mathrm{MHz}$ with the 26-m Penticton radio telescope and with the 46-m dish at the Algonquin Radio Observatory and found that the CPD was consistent with the power-law exponent of -2.5 for the main pulse and with the exponent for the interpulse events equal to -2.8 .

Recently Cordes et al. (2004) in their multifrequency study of the Crab pulsar's giant pulses presented histograms of GP peak amplitudes (S) at 0.43 and $8.8 \mathrm{GHz}$. The histograms represent the PD functions, i.e. number of events per interval of SNR in logarithmic binning $\mathrm{d}(\lg S)$. They found power-law segments in the distributions with the slopes -2.3 and -2.9 at 0.43 and $8.8 \mathrm{GHz}$, respectively. To compare this result with our study of the CPD functions one has to convert the number of events presented by Cordes et al. (2004) from logarithmic binning to linear binning $\mathrm{d}(\lg S)=\ln 10 \mathrm{~d} S / S$. Then, for a power-law fit with linear binning the slope will change from $-\beta$ to $-(\beta+1)$ :

$N(S) \propto(S)^{-\beta} \mathrm{d}(\lg S) \propto(S)^{-(\beta+1)} \mathrm{d} S$ for the power-law fit of the CPD $\gamma=\beta-1$, as was explained in Sect. 3. Thus, the values of the power-law exponent $\beta$ found by Cordes et al. (2004) in their histograms may by immediately compared with our values of the exponents $\gamma$ for the power-law fit of the CPDs. In fact the inspection of the histogram of Cordes et al. (2004) for $0.43 \mathrm{GHz}$ has enabled us to distinguish a break at an $S / N$ value of about 30 where the slope changes from -2.3 to -0.7 in the peak amplitude distribution of the main pulse GPs, while the distribution for the interpulse GPs does not manifest such a break. With the given equivalent flux system temperature of $1262 \mathrm{Jy}$, the receiver band of $12.5 \mathrm{MHz}$, and a sample interval of $128 \mu \mathrm{s}$, the $S / N=30$ break point at $0.43 \mathrm{GHz}$ will correspond to a pulse energy of about $120000 \mathrm{Jy} \mu \mathrm{s}$.

In his Ph.D. thesis Moffett (1997) presentes the CPD functions at $1.4 \mathrm{GHz}$ based on observations conducted with the VLA with a time resolution of $160 \mu$ s. In Fig. 4.3 (page 62) Moffett distinguishes a break in power-law fit of the CPD for the main pulse GPs at a level of 12 Jy corresponding to a pulse energy of about $2000 \mathrm{Jy} \mu \mathrm{s}$, where the slope changes from -3.0 to -1.8 when going from the high flux densities to the lower. Again, the CPD for the GPs originated at the longitudes of the interpulse (Fig. 4.4) does not show a break going straight with the slope of -1.7 until it merges with noise at a level of about $6 \mathrm{Jy}$.

Comparing the values of break-point pulse energy (BPPE) $120000 \mathrm{Jy} \mu \mathrm{s}$ at a frequency of $0.43 \mathrm{GHz}$ (Cordes et al. 2004), $5000 \mathrm{Jy} \mu \mathrm{s}$ at a frequency of $1200 \mathrm{MHz}$ (this paper), and $2000 \mathrm{Jy} \mu$ s at a frequency of $1400 \mathrm{MHz}$ (Moffett 1997), we have found that the BPPE follows a simple power-law frequency dependence $B P P E(v) \approx 7(v)^{-3.4} \mathrm{kJy} \mu$ s, with the exponent -3.4 close to the mean spectral index for the main pulse component of the average profile -3.0 (Moffett 1997). The conclusion can be considered as support for the suggestion that all emission in the main component consists entirely of giant pulses (Popov et al. 2006).

Lundgren et al. (1995) has collected about 30000 GPs from the Crab pulsar at $812 \mathrm{MHz}$ with the Green Bank 43-m radio telescope in 10 days of observations simultaneous with the Compton Gamma Ray Observatory (CGRO) in May 1991. They did not distinguish GPs belonging to the main pulse and the interpulse. Their flux-density distribution was fitted with a powerlaw function for $S>200 \mathrm{Jy}$, and the exponent was found to be equal to -3.46 , the exponent being equal to -2.46 for the CPD used in our analysis. The BPPE value is expected to be $14 \mathrm{kJy} \mu \mathrm{s}$ at $812 \mathrm{MHz}$ according to the frequency relation we derive above. The value corresponds to $50 \mathrm{Jy}$ flux density with $307.5 \mu$ s averaging time used by (Lundgren et al. 1995), and it is well below their threshold of $120 \mathrm{Jy}$. The dramatic roll-off at the rather high pulse energy of about $200 \mathrm{Jy} \times 300 \mu \mathrm{s}=60 \mathrm{kJy} \mu$ s found by (Lundgren et al. 1995) was not observed in the CPDs at 430, 1200 , and $1400 \mathrm{MHz}$ discussed above. It was not observed in the CPD at $600 \mathrm{MHz}$ either, which goes straight with the slope of -2.2 at least down to a pulse energy of about $30 \mathrm{kJy} \mu$ s (Popov et al. 2006). Therefore, it is difficult to reconcile their results with those from the many independent data sets mentioned above.

The power-law form of the observed CPDs can be compared with the field statistics of possible emission mechanisms responsible for the generation of GPs, as discussed by Cairns (2004) who used the values of the exponents of PD functions for comparison. The very short-time flux density variations are of particular interest, since they are closely tied to the physics of the emission process. The power-law indices $\gamma$ of the CPDs at high energies for the shortest GPs are similar enough for both the Crab pulsar and the PSR B $1937+21$, and the values were found to be in the range 1.4 to 1.8 . Converting to indices of 
the probability distribution function (PD) $\beta=\gamma+1$ gives the range $2.4-2.8$ for the values of $\beta$. According to the normalization conditions used by Cairns, the exponent $\alpha$ of the PD of the field $\left(P(\mathcal{E}) \propto \mathcal{E}^{-\alpha}\right)$ is connected with the exponent $\beta$ of the observed PD power-law fit by the relation $\alpha=2 \beta-1$, giving us the range of $\alpha$ from 3.8 to 4.6 to be compared with the theoretical predictions of the field statistics determined by the emission mechanism and propagation effects. The observed breaks in the slope of the CPD functions at certain energies have to be included in theoretical explanations, giving us an extra parameter to constrain the source physics and emission mechanism.

The break in the slope of the CPD functions has important consequences for the estimation of the total rate of GP generation. Popov et al. (2006) made such an estimation for the Crab pulsar under the suggestion that radio emission in the main pulse and in the interpulse consists entirely of giant pulses. They found that about 10 giant pulses are generated during one rotation period of the neutron star. In the estimation they considered that pulse energies of the GPs follow a power-law function with the exponent of -2.2 down to the threshold of about $100 \mathrm{Jy}$ in the peak flux density at a frequency of $600 \mathrm{MHz}$, and with the threshold considered as a real lower limit equivalent to the minimum pulse energy of about $5000 \mathrm{Jy} \mu \mathrm{s}$. With their threshold of about $20000 \mathrm{Jy} \mu$ s in GP detection, they did not notice the break in the CPD slope. The break from -2.2 at high energies to -1.2 at low energies will notably change the estimate of the lower limit for GP energies to about $1000 \mathrm{Jy} \mu$ s, and the rate of GP generation will increase at least by a factor of 2 . The lower energy limit for GP generation, if it exists, will serve as a crucial constraint on the physics of the emission mechanism. To solve the problem, it is necessary to test the low-intensity portion of the CPD function with better sensitivity. Such a study would be possible using VLA or GMRT observations in phased array mode, thereby, significantly reducing the impact from the Crab Nebula. A notable increase in the recording band from 10 to $160 \mathrm{MHz}$ recently achieved for the PuMa II recording systems also makes new observations with the WSRT very promising.

Finally, we summarize the main results of our analysis:

1. The CPDs were found to be notably different for the GPs detected at the longitudes of the main pulse and the interpulse. We suppose that the difference can be explained by the simple attenuation caused by a beaming factor.

2. For the main pulse longitudes, the CPD are different for the GPs of different effective widths with breaks in the CPD power-law indices indicating steepening at high energies (see Table 1).

3. The CPD power-law indices $\left(\gamma_{\text {high }} \approx 1.7\right)$ for the group of short GPs for the Crab pulsar $\left(W_{\mathrm{e}}<10 \mu \mathrm{s}\right)$ are close to the value observed for the millisecond pulsar B1937+21, which seems to generate only the shortest GPs;

4. GPs with a stronger peak flux density were found to be of shorter duration.
The last of these properties testifies in favor of nonlinear temporal models that suggest that the higher the intensity, the narrower the pulse width. Such an emission model was considered, for example, by Mikhailovskii et al. (1985), who treated the micropulses as solitons of the radio-wave envelope propagating through the magnetospheric plasma of the pulsar.

Acknowledgements. This investigation was supported in part by the Russian Foundation for Basic Research (project number 07-02-00074).

\section{References}

Allen, C. W. 1973, Astrophysical quantities (University of London: Athlone Press)

Argyle, E., \& Gower, J. F. R. 1972, ApJ, 175, L89

Backer, D. C. 1971, Ph.D. Thesis, Cornell Univ., Ithaca, NY

Bietenholz, M. F., Kassim, N., Frail, D. A., et al. 1997, ApJ, 490, 291

Cairns, I. H. 2004, ApJ, 610, 948

Cognard, I., Shrauner, J. A., Taylor, J. H., \& Thorsett, S. E. 1996, ApJ, 457, L81

Cordes, J. M., Bhat, N. D. R., Hankins, T. H., McLaughlin, M. A., \& Kern, J. 2004, ApJ, 612, 375

Cusumano, G., Hermsen, W., Kramer, M., et al. 2003, A\&A, 410, L9

Ershov, A. A., \& Kuzmin, A. D. 2003, AstL, 29, 91, transl. from: PAZh, 29, 111 Hankins, T. H. 1971, ApJ, 169, 487

Hankins, T. H. 2000, in Pulsar Astronomy - 2000 and beyond, ed. M. Kramer, N. Wex, \& R. Wielebinski (San Francisco: ASP), ASP Conf. Ser., 202, 165

Hankins, T. H., \& Rickett, B. J. 1975, in Methods in Computational Physics: Advances in Research and Applications, Radio Astronomy, ed. B. Alder, S. Fernbach, \& M. Rotenberg (New York: Academic Press, Inc.), 14, 55

Hankins, T. H., Kern, J. S., Weatherall, J. C., \& Eilek, J. A. 2003, Nature, 422, 141

Hesse, K. H., \& Wielebinski, R. 1974, A\&A, 31, 409

Jessner, A., Slowikowska, A., Klein, B., et al. 2005, AdSpR, 35, 1166

Johnston, S. \& Romani, R. W. 2002, MNRAS, 332, 109

Johnston, S., Van Straten, W., Kramer, M., \& Bailes, M. 2001, ApJ, 549, L101

Johnston, S., Romani, R. W., Roger, W., Marshall, F. E., \& Zhang, W. 2004, MNRAS, 355, 31

Kinkhabwala, A., \& Thorsett, S. E. 2000, ApJ, 535, 365

Knight, H. S., Bailes, M., Manchester, R. N., Ord, S. M., \& Jacoby, B. A. 2006, ApJ, 640, 941

Kramer, M., Johnston, S., \& Van Straten, W. 2002, MNRAS, 334, 523

Kuzmin, A. D., Kondrat'ev, V. I., Kostyuk, S. V., et al. 2002, AstL, 28, 251, transl. from: PAZh, 28, 292

Kuzmin, A. D., Ershov, A. A., \& Losovsky, B. Y. 2004, AstL, 30, 247, transl. from: PAZh, 30, 285

Lundgren, S. C., Cordes, J. M., Ulmer, M., et al. 1995, ApJ, 453, 433

Lyne, A. G. 1982, Jodrell Bank Crab Pulsar Monthly Ephemeris (http://www.jb.man.ac.uk/ pulsar/crab.html)

Mikhailovskii, A. B., Onishchenko, O., \& Smolyakov, A. I. 1985, SvAL, 11, 78, transl. from: PAZh, 11, 190

Moffett, D. A. \& Hankins, T. H. 1996, ApJ, 468, 779

Moffett, D. A. 1997, Ph.D. Thesis, New Mexico Inst. Mining and Technology

Popov, M. V., Soglasnov, V. A., Kondratiev, V. I., et al. 2006, ARep, 83, 660, transl. from: AZh, 83, 717

Ritchings, R. T. 1976, MNRAS, 176, 249

Romani, R. W., \& Johnston, S. 2001, ApJ, 557, L93

Soglasnov, V. A., Popov, M. V., Bartel, N., et al. 2004, ApJ, 616, 439

Voute, J. L. L., Kouwenhoven, M. L. A., Langerak, J. J., et al. 2002, A\&A, 385, 733 